\title{
JCE - a New Perspective for Cardiovascular Emergencies
}

\section{Imre Benedek}

Editor-in-Chief

The Journal of Cardiovascular Emergencies is a new journal in the field of acute cardiac care, dedicated to improving the knowledge and disseminating the results of research related to acute cardiovascular conditions. As Editor-inChief, I have the special honor to launch a journal resulting from a sustained effort of a regional team of experts in cardiovascular emergencies.

Cardiovascular diseases continue to exhibit the highest mortality and morbidity rate in Europe, and Eastern European countries present the highest number of Acute Coronary Syndromes, this situation being associated with still insufficient numbers of patients referred in time for life-saving primary percutaneous interventions [1]. Therefore a regional approach, dedicated to improve the current status of acute cardiac healthcare, based on a synergistic effort coming from key members of cardiovascular professional community in this area, is obviously required. The Journal of Cardiovascular Emergencies aims to bring a significant contribution to strengthen the research potential in this region, from the cardiovascular perspective, by publishing relevant data and top-quality research results of studies dedicated to achieve a better understanding of acute cardiovascular conditions.

In cardiovascular emergencies, and especially in Acute Myocardial Infarctions, a careful attention should be paid to the proper organization of networks that could ensure a fast arrival of the patients to the catheterisation laboratories [2]. Regional models of care for ST-elevation Myocardial Infarction (STEMI), based on regional networks called STEMI networks, have been introduced and studied in different European regions, their functionality and particularity being directly correlated with the regional specifics. The Journal of Cardiovascular Emergencies is the initiative of a group of experts active in the field of organization of regional networks for cardiovascular care, especially in the region of Central-Eastern Europe, a region in which substantial efforts have been made in recent years to improve the access of a large number of patients to a proper treatment. From this perspective, the journal will try to provide the entire cardiology community with the recent advances encountered in the regional cardiology networks in Central-Eastern European countries, reflecting the regional specifics in the care of acute cardiovascular conditions.

However, the journal aims to publish top quality papers related to acute conditions in any cardiovascular pathology, not being restricted to acute coronary syndromes. The subjects covered will focus on recent advances in the field of diagnosis and treatment of the most common causes of cardiovascular emergencies, including acute heart failure, acute aortic diseases, pulmonary embolism, peripheral arterial diseases or cardiac arrhythmia. Interdisciplinary approaches will be also taken into consideration, presenting new advances in the diagnosis and treatment of different other pathologies (i.e. stroke) from a cardiovascular perspective. The Journal of Cardiovascular Emergencies also aims to integrate clinical studies with the pre-clinical work dedicated to the discovery of new mechanisms involved in the development and progression of acute cardiovascular conditions.

On behalf of the editorial board, I would like to invite all the members of acute cardiovascular community to send their research results to be published in the Journal of Cardiovascular Emergencies, a journal dedicated to improve the understanding, the treatment and the survival in acute cardiovascular diseases. 


\section{REFERENCES}

1. Nichols M, Townsend N, Luengo-Fernandez R, Leal J, Gray A, Scarborough P, Rayner M. European Cardiovascular Disease Statistics 2012. European Heart Network, Brussels: European Society of Cardiology, Sophia Antipolis. 2012.

2. Benedek I, Gyongyosi M, Benedek T. A prospective regional registry of ST-elevation myocardial infarction in Central Romania: impact of the Stent for Life Initiative recommendations on patient outcomes. Am Heart J 2013;166:457-465. 